\title{
Comparaisons de diverses méthodes de dénombrement de la flore acidifiante du lait cru
}

par

\author{
J. F. CHAMBA*, Ginette BONNAZ* et P. BOURG **
}

\section{GENERALITES}

Depuis toujours, les fromagers de Gruyère ont apporté une attention particulière à la qualité bactériologique du lait et sa livraison biquotidienne qui existe encore dans de nombreuses fromageries artisanales du Jura et des Alpes a pour objectif de réceptionner des laits aussi peu contaminés que possible. Cependant, cette exigence ne concerne pas seulement l'importance de la contamination bactérienne, mais également la qualité, c'est-à-dire la nature de celle-ci. Or, la composition de la flore du lait est influencée par les conditions de production, de récolte et de conservation. Si de nombreux travaux sur ces influences se sont intéressés à une flore indésirable, tels que les coliformes, les psychrotrophes et les thermorésistants, il y a peu d'études sur la flore acidifiante du lait cru que le fromager considère comme plutôt désirable à condition qu'elle reste à un niveau acceptable.

L'Institut Technique du Gruyère souhaitait entreprendre des études sur ce sujet, mais il était nécessaire de posséder une méthode de dénombrement de la flore acidifiante du lait cru. Il n'existait pas de milieu permettant à lui seul de dénombrer spécifiquement cette flore car elle est constituée d'un grand nombre de bactéries très différentes au point de vue taxonomique. En fait, nous souhaitions pouvoir dénombrer l'ensemble des bactéries présentes dans le lait cru qui sont capables de l'acidifier. Cette flore est essentiellement constituée par des bactéries de type lactique et par ce que Gorrini [6] appelait les acido-protéolytiques dont la majeure partie sont des microcoques.

\footnotetext{
* Institut Technique du Gruyère, zone sud - 74800 La Roche-sur-Foron.

** Ecole Nationale des Industries du lait et des viandes - 74800 La Roche-sur-Foron.
} 
C'est donc essentiellement en fonction du caractère biochimique "production d'acide " que nous avons orienté ce travail.

\section{RECHERCHE D'UNE TECHNIQUE DE DENOMBREMENT}

Nous recherchions un (ou des) milieu de culture permettant une bonne croissance de la flore à dénombrer, mettant en évidence l'acidification produite par la colonie et sur lequel la pousse de la flore non acidifiante soit limitée.

\subsection{Milieux de culture}

La bibliographie ainsi que l'usage en microbiologie laitière [15] nous ont conduit à envisager les milieux suivants :

- Plate Count Agar [2], cette gélose permet la pousse de très nombreuses espèces bactériennes du lait, c'est d'ailleurs la raison pour laquelle elle est largement utilisée pour le dénombrement de la flore mésophile aérobie. Cependant, elle n'est pas très sélective et les ferments lactiques n'y poussent pas très abondamment.

- Milieu d'Elliker [5], est généralement considéré comme excellent pour la croissance de tous les éléments lactiques et les microcoques du lait cru s'y développent également [12].

\subsection{Indicateur d'acidification}

Notre choix s'est porté sur le pourpre de bromocrésol qui est violet-pourpre à $\mathrm{pH} 6,8$ et jaune à $\mathrm{pH} 5,2$ en passant par le vert. Cet indicateur est déjà utilisé en microbiologie (gélose au pourpre) et à un virage assez net à une valeur de $\mathrm{pH}$ convenant bien à notre objectif.

Il est ajouté aux milieux à la dose de $0,025 \mathrm{~g}$ par litre comme dans la gélose au pourpre [2].

\subsection{Agent sélectif}

Nous avons retenu l'acétate de thallium et nous avions le choix entre deux doses, soit 0,05 p. 100 utilisé pour le dénombrement de streptocoques par Roman Pinana [14] qui utilisait ainsi les travaux de Hirsch, Mc Clintock et Mocquot [7], soit 0,1 p. 100 comme dans le milieu de Barnes [2, 9]. Nous avons finalement choisi la dose de 0,1 p. 100 car plus sélective, elle inhibe de nombreuses bactéries dont les coliformes mais permet la croissance de streptocoques lactiques mésophiles et thermophiles, des lactobacilles et, bien sûr, celle des entérocoques mais aussi des leuconostocs et des pédiocoques [11]. 


\subsection{Incubation sous $\mathrm{CO}_{2}$}

De nombreuses bactéries lactiques se développent mieux dans une atmosphère enrichie en $\mathrm{CO}_{2}[11,8]$, nous avons donc essayé l'incubation en jarre avec $\mathrm{CO}_{2}$ (système gaspack).

\subsection{Température et durée d'incubation}

Après quelques essais préalables, nous avons retenu la température de $31^{\circ} \mathrm{C}$ car elle permet la croissance des bactéries lactiques mésophiles, de certains thermophiles et des enterocoques. De plus, cette température réduit, par rapport à $37^{\circ} \mathrm{C}$, la pousse d'une flore parasite non acidifiante. Compte tenu de la température retenue, nous avons incubé pendant $72 \mathrm{~h}$.

\section{MATERIEL ET METHODES}

\subsection{Principe de l'étude}

Nous avons comparé quatre méthodes de dénombrement :

- P.C.A. + pourpre de bromocrésol (P.C.A.P.) ;

- Elliker + pourpre de bromocrésol (E.G.P.) ;

- Elliker + pourpre de bromocrésol + acétate de thallium (E.G.P. th) ;

- Elliker + pourpre de bromocrésol + acétate de thallium avec incubation sous $\mathrm{CO}_{2}$ (E.G.P. th/ $\mathrm{CO}_{2}$ ).

Sur les trois points suivants :

- le dénombrement avec les quatre méthodes à partir des mêmes dilutions de chacun des échantillons afin de comparer les nombres obtenus ;

- sur une trentaine d'échantillons, il a été fait, avec chaque méthode, une détermination en double, afin d'en établir et d'en comparer la répétabilité (dilution en double) ;

- sur chacun des milieux de culture, on a prélevé des colonies qui sont identifiées par une méthode simplifiée $[3,4]$ afin de déterminer la composition bactérienne de la flore dénombrée comme acidifiante, d'en vérifier l'exactitude et de les comparer (voir schéma).

\subsection{Echantillons de lait}

Une centaine d'échantillons ont été choisis de telle sorte que différentes natures de lait : lait frais apporté biquotidiennement à la 


\section{METHODE SIMPLIFIEE D'IDENTIFICATION}

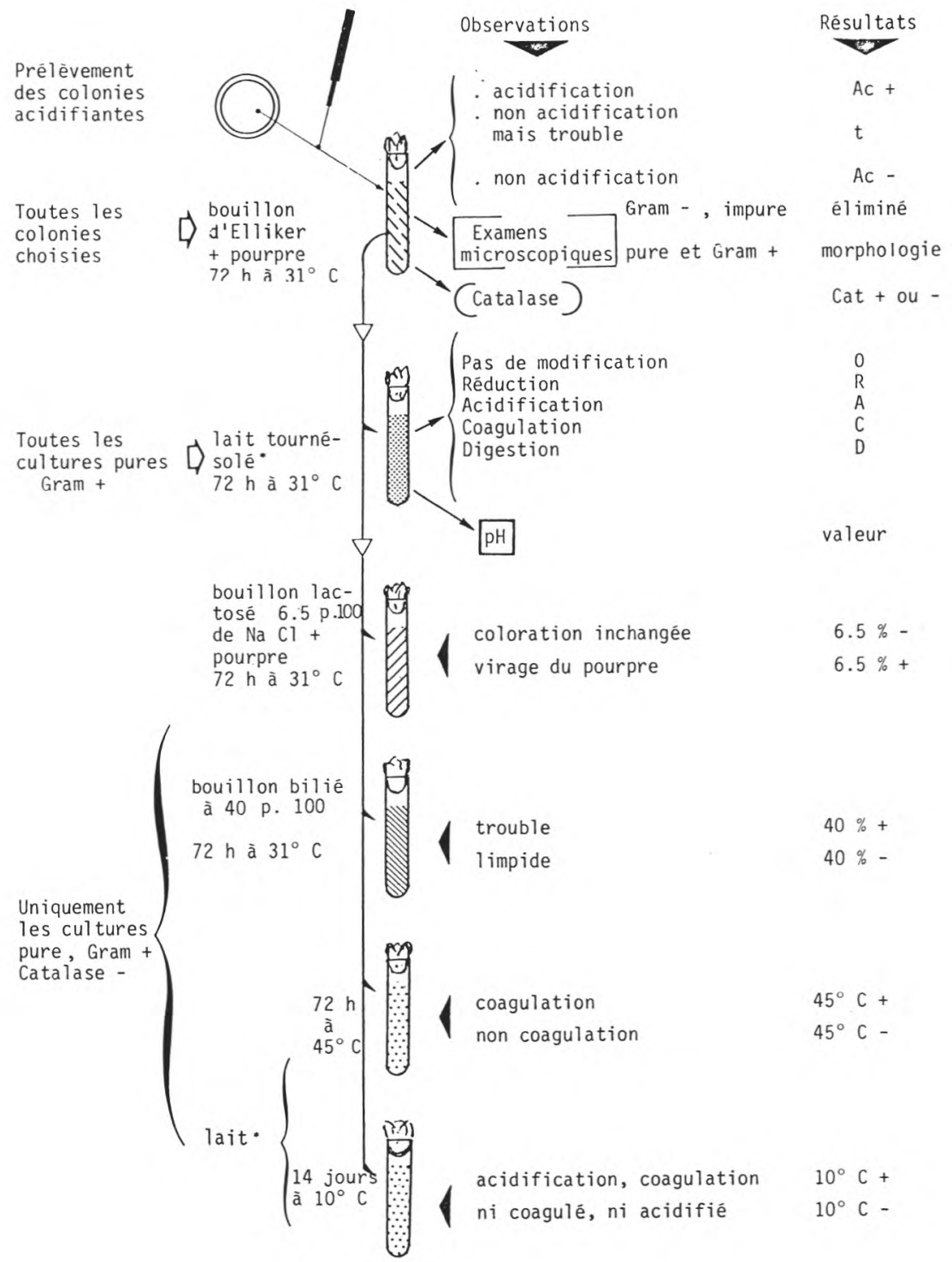

* Le lait écrémé utilisé est obtenu par reconstitution à raison de $100 \mathrm{~g} / \mathrm{l}$ d'une poudre Spray de qualité A garantie sans antibiotique, et dont le $\mathrm{pH}$ après stérilisation est compris entre 6,5 et 6,6 . 
fromagerie, lait refroidi et stocké à la ferme pendant $24 \mathrm{~h}$ et $48 \mathrm{~h}$, ainsi que les différents niveaux de qualité soient représentés.

\section{RESULTATS}

Nous avons travaillé sur 147 échantillons de lait. Les résultats de quelques-uns n'ont pu être utilisés car les boîtes étaient incomptables. Nous possédons les résultats de 109 échantillons qui ont été analysés simultanément avec les quatre techniques.

Dans la plupart des analyses statistiques nous n'avons pas travaillé avec les nombres de bactéries par $\mathrm{ml}$, mais avec leurs logarithmes décimaux. En effet, sans cette transformation, on obtient, compte tenu de la nature de notre échantillon (de quelques milliers à plusieurs millions d'acidifiants $/ \mathrm{ml}$ ), une variance excessive.

\subsection{Aspect des boîtes de Pétri ensemencées et contenant les diffé- rents milieux}

Sur P.C.A.P. les colonies acidifiantes apparaissent parfois assez rapidement, mais souvent la zone de virage du pourpre est assez étendue et il devient alors assez difficile de distinguer les colonies réellement acidifiantes des autres. Dans d'autres cas, des colonies alcalinisantes masquent rapidement la zone virée des colonies acidifiantes voisines. Enfin, on observe des colonies qui acidifient le milieu après $24 \mathrm{~h}$ d'incubation et qui l'alcalinisent ensuite.

De plus, les colonies acidifiantes étant généralement minoritaires parmi l'ensemble des colonies, la lecture est malaisée.

Dans tous les cas, il est nécessaire de faire un pointage des colonies positives après $24 \mathrm{~h}, 48 \mathrm{~h}$ et $72 \mathrm{~h}$ d'incubation.

Avec E.G.P. les problèmes rencontrés sont les mêmes, cependant, les colonies acidifiantes sont plus nettes et plus grosses. Avec E.G.P. $+0,10$ p.100 d'acétate de thallium, les colonies acidifiantes croissant rapidement sont nettes et majoritaires, les colonies parasites non acidifiantes sont très peu nombreuses. L'incubation sous $\mathrm{CO}_{2}$ ne modifie guère l'aspect des boîtes de Pétri contenant E.G.P. th.

\subsection{Evolution du nombre d'acidifiants au cours de l'incubation}

A l'aide de couleurs différentes, nous avons pointé les colonies acidifiantes après 24 h, 48 h et 72 h d'incubation. Alors que le nombre d'acidifiants augmente très rapidement tout au long de l'incubation sur E.G.P. th, on observe, sur 137 dénombrements une diminution apparente du nombre d'acidifiants dans 25,8 p. 100 des cas entre 48 et $72 \mathrm{~h}$ avec P.C.A.P. et de 15,7 p. 100 avec E.G.P. du fait du développement de colonies alcalinisantes. 


\subsection{Comparaisons des dénombrements obtenus avec chaque méthode}

Elles portent sur les dénombrements effectués après $72 \mathrm{~h}$ d'incubation sur les 109 échantillons de lait cru analysés simultanément. Les moyennes sont en log décimaux et le test employé est celui du t des observations appariées.

\section{TABLEAU 1}

Comparaisons des méthodes de dénombrement

\begin{tabular}{l|c|c|c}
\hline \multicolumn{1}{c|}{ Méthodes } & Moyenne & \multicolumn{1}{c}{ Signification des différences } \\
\cline { 2 - 3 } P.C.A.P. & 4,357 & $\begin{array}{l}\text { probablement } \\
\text { significative } \\
0,95<\mathrm{P}<0,99\end{array}$ & significatives \\
E.G.P. & 4,653 & $\begin{array}{l}\text { non significative } \\
\mathrm{P}<0,95\end{array}$ & $\mathrm{P}>0,99$ \\
E.G.P. th & 4,491 & & \\
E.G.P. th $/ \mathrm{CO}_{2}$ & 4,492 &
\end{tabular}

Les conclusions que l'on peut tirer de ces comparaisons sont les suivantes :

le P.C.A. ; milieu d'Elliker permet de dénombrer plus d'acidifiants que
le

- l'addition de 0,1 p. 100 d'acétate de thallium conduit à une réduction de la flore acidifiante dénombrée ;

- l'incubation sous $\mathrm{CO}_{2}$ ne semble présenter aucun intérêt du point de vue du dénombrement.

\subsection{La répétabilité de chacune des méthodes de dénombrement}

Dans les conditions expérimentales de cette étude, elle est définie comme l'écart entre deux résultats provenant du même opérateur, qui, dans une longue série d'essais (ici 31) ne serait dépassée que dans 5 cas sur 100 [1].

Plus la répétabilité, c'est-à-dire l'écart entre les deux résultats, aura une faible valeur, plus la fidélité de la méthode employée sera bonne. 
TABLEAU 2

Répétabilité des méthodes de dénombrement

\begin{tabular}{l|c|c|c|c}
\hline \multicolumn{1}{c|}{ Milieux } & P.C.A.P. & E.G.P. & E.G.P. th & E.G.P. th $/ \mathrm{CO}_{2}$ \\
\cline { 1 - 2 } Moyenne & 4,273 & 4,624 & 4,383 & 4,382 \\
Ecart moyen & 0,246 & 0,130 & 0,065 & 0,087 \\
$\begin{array}{l}\text { Nombre des valeurs } \\
\text { éliminées* }\end{array}$ & 1 & 1 & 0 & 1 \\
Répétabilité & 0,720 & 0,319 & 0,174 & 0,232 \\
\hline
\end{tabular}

* Par le test des limites (1).

Ainsi, la répétabilité est bien meilleure sur E.G.P. que sur P.C.A.P. L'addition de 1 p. 1000 d'acétate de thallium améliore nettement la répétabilité du dénombrement alors que l'incubation sous $\mathrm{CO}_{2}$ la dégrade légèrement.

\subsection{Identification simplifiée de la flore acidifiante}

4.5.1. Caractères des colonies isolées.

Dans le tableau 3 sont indiqués :

- les colonies utiles : ni impures, ni Gram - ;

- les colonies fausses positives : elles n'ont pas été capables d'acidifier le bouillon d'Elliker + pourpre en $72 \mathrm{~h}$ à $31^{\circ} \mathrm{C}$, en p. 100 des colonies utiles ;

- les caractéristiques des colonies acidifiantes : catalase et réduction de lait tournesolé, $\mathrm{pH}$ obtenu sur lait tournesolé après $72 \mathrm{~h}$ à $31^{\circ} \mathrm{C}$; en p. 100 des colonies utiles.

Nous avons choisi 5,50 comme limite de $\mathrm{pH}$ pour départager les colonies nettement acidifiantes des autres, c'est-à-dire un abaissement du $\mathrm{pH}$ du lait de 1 point en $72 \mathrm{~h}$ à $31^{\circ} \mathrm{C}$.

La comparaison à l'aide du test du $\chi^{2}$ des proportions colonies acidifiantes/colonies utiles indique une différence significative $(\mathrm{P}>0,99)$ pour l'ensemble des milieux, la comparaison des proportions deux par deux indique que P.C.A.P. et E.G.P. ainsi que P.C.A.P. et E.G.P. th sont différents (P>0,95 p. 100), alors qu'il n'y a pas de différence entre E.G.P. et E.G.P. th ainsi qu'entre E.G.P. th et E.G.P. th $/ \mathrm{CO}_{2}$.

Les calculs effectués avec les proportions colonies acidifiantes catalase-/colonies utiles, donnent exactement les mêmes résultats. 


\section{TABLEAU 3}

Les colonies isolées sur les différents milieux de culture

\begin{tabular}{|c|c|c|c|c|c|c|c|}
\hline \multirow{3}{*}{$\begin{array}{l}\text { Milieux } \\
\text { de } \\
\text { culture }\end{array}$} & \multirow{3}{*}{$\begin{array}{c}\text { Nombre } \\
\text { de } \\
\text { colonies } \\
\text { utiles }\end{array}$} & \multicolumn{6}{|c|}{ En p. 100 des colonies utiles } \\
\hline & & \multirow{2}{*}{$\begin{array}{l}\text { Fausses } \\
\text { positives }\end{array}$} & \multirow{2}{*}{$\begin{array}{l}\text { Réduc- } \\
\text { tion du } \\
\text { lait tour- } \\
\text { nesolé }\end{array}$} & \multicolumn{2}{|c|}{$\begin{array}{c}\text { Acidification } \\
\mathrm{pH}<5,50\end{array}$} & \multicolumn{2}{|c|}{$\mathrm{pH}>5,50$} \\
\hline & & & & Catalase & $\begin{array}{c}\text { Catalase } \\
+\end{array}$ & Catalase & $\begin{array}{c}\text { Catalase } \\
+\end{array}$ \\
\hline P.C.A.P. & 98 & 19,3 & 41,1 & 42,8 & 13,3 & 22,4 & 21,5 \\
\hline E.G.P. & 99 & 11,1 & 51,8 & 60,6 & 13,1 & 11,1 & 15,2 \\
\hline E.G.P. th & 99 & 5,0 & 63,6 & 72,7 & 8,1 & 12,1 & 7,1 \\
\hline E.G.P. th $/ \mathrm{CO}_{2}$ & 99 & 8,1 & 65,4 & 66,6 & 10,1 & 14,2 & 9,1 \\
\hline
\end{tabular}

\subsubsection{Nature des acidifiants isolés}

Dans le tableau 4, on trouvera la nature de toutes colonies catalase - capables d'acidifier le lait tournesolé à un $\mathrm{pH}$ inférieur à 5,50 .

Tout d'abord, il apparaît que le milieu d'Elliker est nettement plus favorable à la pousse de la flore lactique que le P.C.A. ce à quoi nous nous attendions.

L'addition de 0,1 p. 100 d'acétate de thallium modifie quelque peu la composition de la flore acidifiante. En effet, avec le milieu E.G.P. th, on trouve plus de S. lactiques mésophiles et de S. sp. qu'avec E.G.P. L'absence de bacilles sur le milieu E.G.P. th doit être interprétée avec prudence compte tenu de la rareté de ce groupe dans les échantillons de lait analysés.

L'incubation sous $\mathrm{CO}_{2}$ se révèle favorable pour les $S$. thermophilus puisqu'on le trouve presque deux fois plus souvent que lorsque l'incubation a lieu en atmosphère normale.

On voit alors que les différents groupes sont représentés dans des proportions assez voisines dans chacun des milieux. En effet, la comparaison des proportions de chaque groupe avec le test du $\chi^{2}$ ne permet pour aucun des groupes de streptocoques de conclure à l'existence d'une différence entre les milieux. Il en est de même pour les bacilles. 
TABLEAU 4

Identification simplifiée des colonies acidifiantes, catalase - en p. 100

\begin{tabular}{l|c|c|c|c|c}
\hline \multirow{2}{*}{\multicolumn{1}{c|}{ Milieux }} & \multicolumn{4}{|c|}{ Streptocoques } & \multirow{2}{*}{ Bacilles } \\
\cline { 2 - 5 } & mésophiles & thermophiles & fécaux & s.p. & \\
\cline { 3 - 5 } & 35,7 & 11,9 & 11,9 & 31,8 & 2,4 \\
\cline { 3 - 5 } P.C.A.P. & 38,3 & 10,0 & 15,0 & 31,7 & 5,0 \\
E.G.P. & 45,8 & 8,3 & 9,7 & 36,2 & - \\
E.G.P. th & 39,4 & 15,1 & 9,1 & 36,4 & - \\
E.G.P. th/ $/ \mathrm{CO}_{2}$ & & & &
\end{tabular}

Donc, la composition de la flore acidifiante n'est pas différente d'un milieu à l'autre.

\section{Cas des streptocoques sp.}

Ce groupe rassemble tous les clônes ayant un aspect morphologique en diplocoque ou streptocoque, catalase - , acidifiant le lait à un $\mathrm{pH}$ inférieur à 5,50 , mais dont les autres caractères biochimiques ne permettent pas un classement dans les autres groupes. Ces streptocoques que notre méthode simplifiée ne permet pas d'identifier plus précisément, occupe une place importante dans la flore acidifiante puisque c'est, après les streptocoques mésophiles, celui dont l'effectif est le plus fourni.

Dans quelques rares cas, il peut s'agir soit d'un mélange de streptocoques lactiques mésophiles et de $S$, thermophilus, soit de $S$. uberis (croissance à $10^{\circ} \mathrm{C}$ et $45^{\circ} \mathrm{C}$ mais pas avec $6,5 \mathrm{p} .100$ de $\mathrm{Na} \mathrm{Cl}$ et 40 p. 100 de bile). Pour les autres clônes que nous avons classés dans le groupe de Streptocoques $\mathrm{sp}$, on peut faire diverses hypothèses quant à leur identité $[3,9]$. Il peut s'agir pour une partie de Streptocoques mésophiles croissant mal à $10^{\circ} \mathrm{C}$, de Streptocoques du groupe " pyogène " classés par Prévot [13] dans le groupe "viridans ». Par ailleurs, on peut penser que dans ces indéterminés, nous avons isolé des leuconostocs et des pédiocoques.

Enfin, dans le Bergey's [3], il est cité des streptocoques dont les caractères sont peu connus et que les auteurs ont classés dans divers groupes de S. sp. Il est très probable que nous ayons isolé de nombreuses colonies de ces streptocoques indéterminés. 


\section{DISCUSSION - CONCLUSION}

L'ensemble des résultats que nous avons obtenus montrent que le Plate Count Agar additionné de pourpre de bromocresol ne permet pas de dénombrer convenablement la flore acidifiante du lait cru.

Par contre, le milieu d'Elliker additionné de pourpre de bromocresol permet un bon développement de la flore acidifiante, la plus grande richesse du milieu d'Elliker en sucres fermentescibles explique sûrement ce meilleur développement que sur P.C.A. D'ailleurs, cette excellente aptitude du milieu d'Elliker à la croissance des bactéries lactiques thermophiles a été également soulignée par Walter, Hasselmann et Castillo [16]. Cependant, la lecture des boîtes est malaisée et conduit à des erreurs (11 p. 100 de faux positifs), cela est le fait du développement, parfois intense, d'une flore " parasite " non acidifiante voire même alcalanisante.

L'addition de 0,1 p. 100 d'acétate de thallium au milieu d'Elliker permet une lecture beaucoup plus facile et le nombre de colonies faussement positives est diminué de moitié par l'inhibition de la flore " parasite ». Cela améliore la qualité et la précision du dénombrement puisque c'est avec l'acétate de thallium que l'on obtient la meilleure répétabilité, mais cela réduit significativement de l'ordre de 20 p. 100 , le nombre d'acidifiants dénombrés. Cette réduction du nombre de colonies dénombrées comme acidifiantes est, pour une partie : $6 \mathrm{p} .100$ due à la réduction du nombre de faux positifs. Par ailleurs, les colonies acidifiantes sur milieu d'Elliker avec acétate de thallium sont plus fréquemment que sans acétate des colonies de bactéries réellement capables d'acidifier le lait : 80,8 p. 100 au lieu de 73,7 p.100. De plus, la proportion de colonies acidifiantes catalase - est plus forte sur Elliker + acétate : 72,7 p. 100 que sur Elliker : 60,6 p. 100. D'ailleurs, les observations que nous avions faites avec les différentes souches de bactéries lactiques montraient que l'acétate de thallium ne gênait pas la croissance de celles-ci, ce qui est également le cas sur le milieu de Barnes [2] qui, lui aussi, contient 0,1 p. 100 d'acétate de thallium.

Nous trouvons moins de germes catalase + sur E.G.P. th $(15,2$ p. 100$)$ que sur E.G.P. (28,3 p. 100). Ces germes qui sont d'après les examens microscopiques, en majorité des microcoques et des staphylocoques sont rencontrés aussi fréquemment dans nos échantillons de lait que sur les échantillons de lait analysés par Ordonnez et Burgos [12] : entre 25,9 p. 100 et 33,3 p. 100 des germes proliférant sur gélose d'Elliker.

Enfin, on peut remarquer qu'environ la moitié de ces bactéries catalase + sont capables d'acidifier le lait à un $\mathrm{pH}$ inférieur à 5,50 et constituent très certainement ce que GoRRINI [6] appelait les " acido-protéolytiques". 
Par ailleurs, l'incubation sous $\mathrm{CO}_{2}$ n'améliore pas les résultats obtenus avec le milieu d'Elliker + acétate de thallium. On n'obtient pas des nombres plus élevés d'acidifiants, la répétabilité est un peu moins bonne et les colonies dénombrées ne sont pas plus fréquemment des germes capables d'acidifier le lait.

L'identification simplifiée des colonies acidifiantes montre que les proportions des différents streptocoques sont très voisines dans les différents milieux utilisés.

Dans les échantillons analysés, ce sont les streptocoques lactiques (S. lactis, S. cremoris et $S$. diacetilactis) qui sont en plus grand nombre suivis d'un groupe de bactéries capables d'acidifier le lait que la méthode employée ne permet pas d'identifier plus précisément. Ensuite, on trouve dans une proportion voisine, entre 5 et 10 p. 100 des colonies acidifiantes $S$. thermophilus et les streptocoques fécaux. Les bacilles acidifiant sont très rarement rencontrés dans les échantillons de lait que nous avons analysés. Ces résultats ne sont pas très différents de ceux de Maret et Sozzi [10] qui, en utilisant des galeries API modifiées pour l'identification de colonies de ferments lactiques isolés de laits d'alpages suisses, ont surtout trouvé des Streptococcus lactis, des leuconostocs et des entérocoques, mais n'ont $\mathrm{pu}$ isoler des streptocoques thermophiles ni des lactobacilles.

En conclusion, l'utilisation du milieu d'Elliker convient bien au développement de la flore acidifiante, l'addition d'acétate de thallium à la dose de 0,1 p. 100 permet d'en améliorer la sélectivité, d'accroître la justesse et la précision de l'analyse. Les nombres obtenus surestiment la population de bactéries acidifiantes puisque dans les colonies dénombrées comme acidifiantes, 80,8 p. 100 sont capables d'acidifier le lait à un pH inférieur à 5,50 en 72 h. Toutefois, 95 p. 100 des colonies produisent de l'acide en quantité notable (acidification du bouillon d'Elliker) dont 80,8 p. 100 peuvent le faire à partir du lactose et 14,2 p. 100 à partir des autres sucres du milieu d'Elliker (dextrose et saccharose). Il est possible que dans le lait, certaines de ces bactéries qui ne produisent que très peu d'acide à partir du lactose, participent à son acidification lorsqu'elles sont associées aux autres bactéries présentes dans le lait cru.

Certes, nous savions qu'il ne fallait guère espérer que l'utilisation d'un milieu, aussi bien adapté soit-il, permette de dénombrer spécifiquement la flore acidifiante du lait cru car celle-ci est constituée de bactéries très différentes au point de vue taxonomique. Cependant, l'utilisation de la gélose d'Elliker additionnée de 0,1 p. 100 d'acétate de thallium et de 0,025 p. 1000 de pourpre de bromocresol permet une assez bonne estimation du nombre de bactéries acidifiantes du lait cru. Enfin, ce travail nous a permis de mieux cerner la nature des principaux groupes bactériens du lait cru qui sont capables de l'acidifier. 


\title{
Rés u mé
}

Il a été comparé diverses méthodes de dénombrement de la flore acidifiante du lait cru : Plate Count Agar, gélose d'Elliker additionnée ou non de 0,1 p. 100 d'acétate de thallium avec ou sans incubation sous $\mathrm{CO}_{2}$. Le pourpre de bromocresol à la dose de $0,025 \mathrm{~g} / 1$ a été ajouté comme indicateur d'acidification. Ces méthodes ont été employées pour le dénombrement de la flore acidifiante de 147 échantillons de lait d'origine et de qualité variées. Sur chaque milieu, une centaine de colonies acidifiantes ont été isolées et identifiées par une méthode simplifiée.

C'est la gélose d'Elliker additionnée de 0,1 p. 100 d'acétate de thallium et de $0,025 \mathrm{~g} / 1$ de pourpre de bromocresol étuvé à $31^{\circ} \mathrm{C}$ qui permet le meilleur dénombrement de la flore acidifiante du lait cru. L'incubation sous $\mathrm{CO}_{2}$ n'améliore pas la qualité du dénombrement. Enfin, ce travail nous a permis de mieux cerner la nature des principaux groupes bactériens du lait cru qui sont capables de l'acidifier.

\section{S u m m a r y}

\author{
COMPARISONS BETWEEN VARIOUS METHODS \\ TO COUNT THE ACIDIFYING FLORA IN RAW MILK
}

Various methods have been compared to count the acidifying flora in raw milk. Plate Count Agar, Elliker Agar-Agar added or not to $0,1 \mathrm{p} .100$ thallous acetate with or without incubation in $\mathrm{CO}_{2}$. A $0,025 \mathrm{~g} / 1$ of bromocresol purple has been added as an indicator of acidification. Those methods have been used to count the acidifying flora in 147 samples of raw milk which have had various origines and qualities. On each medium near one hundred of acidifying colonies have been isolated and identified with a simplified method.

It's Elliker Agar-Agar added to 0,1 p. 100 thallous acetate and to $0,025 \mathrm{~g} / 1$ of bromocresol purple incubated at $31^{\circ} \mathrm{C}$ wich enable a better way to count the acidifying flora in raw milk. Incubation in $\mathrm{CO}_{2}$ didn't improve the quality of counting. Finally, this work have enable us better define the nature of the main bacterial groups in raw milk capable of acidifying it.

Reçu pour publication en janvier 1981.

\section{Bibliographie}

[1] A.F.N.O.R. (1974). - Recueil des normes statistiques, tome I, $1^{\mathrm{e}}$ édition.

[2] B.B.L. (1968). - Manual of products and laboratory procedures, 5th edition. 
[3] Bergey's manual of determinative bacteriology (1974), 8th edition.

[4] Butriaux (1966). - Manuel de technique bactériologique, $2^{c}$ édition, Ed. Médicales Flammarion.

[5] Elliker (P. A.), Anderson (A. W.) and Hanneson (G.) (1956). - Agar medium for lactic acid bacteria. J. Dairy Science, 39, 1, 611.

[6] GoRrini (C.) (1949). - Les ferments lactiques acidoprotéolytiques. Le Lait, 29, 337-351.

[7] Hirsch (A.), Mc Clintock (M.) and Moceuot (G.) (1952). - Observations on the influence of inhibitory substances produced by the lactobacilli of Gruyère cheese on the development of anaerobie sporeformers. J. Dairy Research, 19, 179-186.

[8] Kanbe (M.) (1974). - Effet du gaz carbonique sur le développement des lactobacilles dans le milieu gélosé - tryptone-glucose-extrait de levure. 19 e Cong. Int. Laiterie, $1 \mathrm{~F}, 427-428$.

[9] Larpent (J. P.) et Larpent-Gourgaud (M.) (1975). - Memento technique microbiologie. Ed. P.S.I. Technique et documentation.

[10] Maret (R.) et Sozzi (T.) (1976). - Flore lactique des fromageries d'alpages suisses. Le Lait, 56, 304-318.

[11] Merck (E.). - Manuel de microbiologie, Ed. Merck.

[12] Ordonnez (J.A.) et Burgos (J.) (1977). - Etude de la variété de fromage Ulloa. Evolution de la flore microbienne et des composants azotés au cours de la maturation. Le Lait, 57, 150-163.

[13] Prévot (A. R.) (1961). - Traité de systématique bactérienne, tome II, Dunod éditeur.

[14] Roman Pinana (M.) (1975). - Etude de la flore microbienne du fromage espagnol Manchego. Le Lait, 55, 401-413.

[15] Serre (S.), Amariglio (S.) et Petransxienne (D.) (1973). - Contrôle de la qualité des produits laitiers. Informations Techniques des Services Vétérinaires.

[16] Walter (J.), Hasselmann (M.) et Castillo (M.) (1978). - Aspects quantitatifs de la flore bactérienne normale des yaourts. Dénombrement, évolution durant la conservation, identification. Rev. Lait. Française, 362, 117-129. 